\section{Discussion}

A weakness of this study was that we were only able to measure the air leaving the lungs due to leaking around the facemask. Although data from our previous study makes it seem unlikely, ${ }^{4}$ some air may have remained in the lungs during the first three breaths and thus we may have underestimated the true tidal exchange. Nevertheless, we consider that the data in this paper indicate that facemask resuscitation rarely provides adequate gaseous exchange on its own. Despite this all the babies studied responded satisfactorily and were breathing within four minutes. During this period adequate tidal exchange appeared to be almost totally due to Head's paradoxical reflexes - that is, the inflation pressure inducing the baby to make inspiratory efforts. This almost always produced tidal exchange considerably in excess of twice the anatomical dead space. The time to the first effective respiratory effort tended to be earlier with facemask resuscitation, suggesting that the process of intubation might delay the onset of respiratory efforts. The time required for resuscitation was overall rather longer with facemask resuscitation, but this was almost entirely due to results from a single baby. The poor ventilatory response to the facemask system appeared to be due to the long time constant of the fluid filled lung, so that the relatively short period of inflation of the Laerdal bag was far too short to overcome the highly viscous forces. ${ }^{3}$ We suspect that increased ventilatory exchange could be achieved by modifying the blow off valve or by using a larger inflation reservoir. This might, however, lead to more air entering the oesophagus and distending the stomach. Our conclusion is that facemask resuscitation is effective if, as often happens, it stimulates reflex inspiratory efforts (Head's reflex). If this response does not occur endotracheal intubation is necessary to produce adequate exchange.

We thank the Medical Research Council for financial support and the midwives and obstetricians of the City Hospital, Nottingham, for their cooperation and encouragement.

\section{References}

1 American Heart Association. Basic life support in infants and children. $7 A M A$ $1980 ; 244: 472-8$.

Vyas $\mathrm{H}$, Milner AD, Hopkin IE. Facemask resuscitation: does it lead to gastric distension? Arch Dis Child 1983;58:373-5.

Vyas $\mathrm{H}$, Milner AD, Hopkin IE. Physiological responses to prolonged and slow rise inflation. F Pediatr 1981;99:635.

4 Vyas $\mathrm{H}$, Milner $\mathrm{AD}$, Hopkin IE. Intrathoracic pressure and volume changes during the spontaneous onset of respiration in babies born by caesarian section and by vaginal deliveries. $\mathcal{F}$ Pediatr $1981 ; 99: 787-91$.

(Accepted 12 September 1984)

\title{
Cutaneous insulin allergy responsive to oral desensitisation and aspirin
}

\author{
I M HOLDAWAY, J D WILSON
}

\begin{abstract}
A diabetic man with no previous history of allergy began to suffer itchy, painful swelling at the sites of injection after three months' treatment with bovine insulin. Insulin specific IgE concentrations $(1 \cdot 2-2.0 \mathrm{U} / \mathrm{ml})$ were higher than in non-allergic diabetics (mean 0.4 (SD 0.06) $\mathrm{U} / \mathrm{ml}$ ) but lower than in most other patients allergic to insulin (1.0-19.0 $\mathrm{U} / \mathrm{ml})$. Standard approaches failed to overcome the allergic reaction, and four separate attempts at desensitisation were unsuccessful.

The patient was then given oral insulin $800 \mathrm{U}$ thrice daily together with enteric coated aspirin $1300 \mathrm{mg}$ thrice daily for one week, and subsequent desensitisation with neutral insulin injection was carried out successfully. On stopping the aspirin the original reactions returned, and aspirin was therefore reinstituted as a permanent part of treatment.
\end{abstract}

Whatever the mechanism in this patient, oral desensitisation and aspirin provided a simple method for treating a difficult condition.

\footnotetext{
Departments of Endocrinology and Immunology, Auckland Hospital and Auckland University School of Medicine, Auckland, New Zealand

I M HOLDAWAY, MD, FRACP, endocrinologist and associate professor of medicine

J D WILSON, MD, FRACP, immunologist and associate professor of medicine

Correspondence to: Dr I M Holdaway, Associate Professor of Medicine, Auckland Hospital, Auckland 1, New Zealand.
}

\section{Introduction}

Allergy to insulin may be local or systemic ${ }^{1}$ and is usually due to antigenicity of non-human insulin, additives, or contaminants. We describe a patient with localised insulin allergy resistant to standard desensitisation regimens who responded to oral desensitisation together with administration of a non-steroidal anti-inflammatory agent.

\section{Case history}

A 37 year old diabetic man presenting with ketosis and weight loss developed cutaneous allergy after three months' treatment with bovine insulin. Coin sized raised, red, itchy swellings developed within 15 minutes of injection, with some larger swellings associated with deep throbbing pain. Often a typical reaction would be associated with itchiness, swelling, and redness at the site of previous injections. The reactions corresponded to an "immediate" type of cutaneous allergy. There was no personal or family history of allergy.

Insulin specific IgE antibodies were measured by adsorption to an insulin-Sepharose column and quantified by reaction with iodine- 125 labelled antihuman IgE. ${ }^{2}$ Mean insulin specific IgE concentrations in the patient ranged from 1.2 to $2.0 \mathrm{U} / \mathrm{ml}$ compared with 1.0 to $19.0 \mathrm{U} / \mathrm{ml}$ in other patients with insulin allergy, $0.6(\mathrm{SD} 0.1) \mathrm{U} / \mathrm{ml}$ in non-diabetics, and $0.4(\mathrm{SD} 0.06) \mathrm{U} / \mathrm{ml}$ in non-allergic diabetics. ${ }^{2}$

Four different approaches were used in an effort to overcome the allergic reaction-namely, reduction in antigenicity of the injected material; desensitisation to reduce allergic sensitivity; use of antagonists to the mediators of the allergic reaction; and prevention of synthesis of allergy mediators. Insulin was withdrawn and the patient treated with restricted diet and oral hypoglycaemic agents during attempts at desensitisation. Bovine or porcine insulin from various manufacturers; highly purified monocomponent porcine, bovine, and human insulin (Novo Industries); desphe insulin (Hoescht); and zinc free sodium insulin (Eli Lilly) all caused brisk 
reactions when given either intradermally or subcutaneously. Insulin carrier media (Novo) did not induce a reaction.

Four separate attempts were made at desensitisation with parenteral insulin using either rapid $^{3}$ or slow ${ }^{4}$ desensitisation schedules, but these were unsuccessful. Antihistamines (chlorpheniramine $4 \mathrm{mg}$ four times daily by mouth or $5 \mathrm{mg}$ subcutaneously with insulin injections; cimetidine $200 \mathrm{mg}$ three times daily by mouth or $100 \mathrm{mg}$ subcutaneously with insulin injections) and dexamethasone $0.5 \mathrm{mg}$ with each injection for three days were without effect. Two days of prednisone $20 \mathrm{mg}$ daily was without benefit. Long term, higher dose steroids were not given because of their likely effect on the diabetes and their unsuitability for long term management.

Finally, desensitisation was attempted using oral insulin together with non-steroidal anti-inflammatory agents to antagonise vascular mediators of the reaction. Regular insulin, $800 \mathrm{U}$ by mouth three times daily before meals, and enteric coated aspirin (Rhusal, Roerig), $1300 \mathrm{mg}$ three times daily, were given for one week. Desensitisation was then carried out successfully using neutral insulin (Actrapid MC). Over the next six months he had very occasional swellings at the site of injection without pain or itch. He then stopped aspirin voluntarily and within a few days the original allergic reactions returned. Aspirin $1300 \mathrm{mg}$ twice daily again reduced reactions to once every one or two weeks and was continued permanently. Diabetic control assessed by home monitoring of capillary blood glucose values was excellent, with mean glucose concentrations of 7-8 mmol/l (126-144 mg/100 ml).

\section{Comment}

Cutaneous allergy to insulin resistant to standard desensitisation measures is rare. ${ }^{1}$ Although desensitisation by parenteral means has been widely used in patients sensitive to inhalant allergens, the oral approach to desensitisation has been much less studied. There is only trivial absorption of oral insulin, and desensitisation by this route may not require systemic absorption of the allergen. In our patient oral desensitisation alone proved insufficient to control the allergy, so that full control and long term remission was achieved only with the addition of aspirin. It is indeed uncertain whether oral desensitisation was necessary. Non-steroidal anti-inflammatory drugs have a broad range of action and might readily affect local vascular changes concerned in the allergic process and so explain the results seen in this patient. Whatever the mechanism, the present approach provided a simple method for treating a difficult clinical condition.

IgE was kindly measured by $\mathrm{K}$ Falholt (Novo Research Laboratories, Copenhagen). We are grateful to Professor John Turtle, the Royal Prince Alfred Hospital, Sydney, Australia, for suggesting the use of oral insulin. This study was supported by the New Zealand Medical Research Council.

\section{References}

1 Galloway JA, Bressler R. Insulin treatment in diabetes. Med Clin North Am $1978 ; 62: 663-71$

2 Falholt $\mathrm{K}$. Determination of insulin specific IgE in serum of diabetic patients by solid-phase radioimmunoassay. Diabetologia $1982 ; 22: 254-7$.

Elenbaas RM, Forni PJ. Management of insulin allergy and resistance. Am $\mathcal{Y}$ Hosp Pharm 1976;33:491-7.

Leiberman $\mathrm{P}$, Patterson $\mathrm{R}$, Metz $\mathrm{R}$, Lucena J. Allergic reactions to insulin. FAMA $1971 ; 215: 1106-12$.

(Accepted 10 October 1984)

\begin{abstract}
One hundred and five children and adolescents with impaired hearing and 19 with impaired vision underwent in vitro tests (lymphocyte responsiveness and serum antibody to rubella) for retrospective diagnosis of intrauterine rubella. Tests yielded results consistent with intrauterine rubella in $30(29 \%)$ of the patients with impaired hearing but only one $(5 \%)$ of those with impaired vision. In addition, the reported incidence $(\mathbf{1 0} \cdot \mathbf{8} \%)$ of rubella as a cause of deafness was obtained by questioning parents before the tests. Of 27 patients with impaired hearing of unknown aetiology but reported rubella contact during the pregnancy, seven $(26 \%)$ had test results consistent with intrauterine rubella.

The incidence of intrauterine rubella as a cause of deafness is probably underestimated when the diagnosis is based on the presence of several classic features.

\footnotetext{
Department of Immunology, Royal Children's Hospital, Melbourne, Victoria 3052, Australia

J L IUORIO, BSC, research scientist

C S HOSKING, MD, FRACP, director

C PYMAN, FRACS, DLO, consultant ENT surgeon

Correspondence to: Dr C S Hosking.
}

\section{Introduction}

For over a century rubella (or German measles) was considered to be a mild disease. Then in 1941 the intrauterine effects of rubella were recognised. ${ }^{1}$ By 1943 maternal rubella during pregnancy was documented as being associated with deafness, blindness, heart disease, and other congenital malformations among infants. ${ }^{2}$ In 1969 Cooper et al $^{3}$ estimated that the incidence of fetal damage after maternal infection in the first trimester of pregnancy was "in excess of $20 \%$." 3 These results were confirmed by Miller et al, ${ }^{4}$ but the numbers in their study were small.

The diagnosis of intrauterine rubella is easy to confirm in infants born with several classic features of the disease.$^{5}$ In other children affected by rubella the clinical diagnosis may be impossible, particularly in those infected later in pregnancy whose only clinical feature may be nerve deafness, which will not be diagnosed in infancy at a time when viral culture can offer confirmation. ${ }^{6}$

In 1979 Buimovici-Klien et al showed that lymphocyte transformation after stimulation with phytohaemagglutinin was significantly lower in children with congenital rubella than in healthy controls. ? Specific lymphocyte responses to purified rubella virus were absent or at least two times lower in children with congenital rubella than in immune controls. In 1981 workers at this laboratory investigated these claims. The results obtained led to the development of a useful diagnostic test, using readily available reagents, to measure the immunological responses to rubella virus antigens in vitro. ${ }^{8}$ Lymphocytes from 